\title{
Baryogenesis from flavon decays
}

\author{
Mu-Chun Chen, Seyda Ipek, and Michael Ratz \\ Department of Physics and Astronomy, University of California, Irvine, California 926974575, USA
}

(Received 22 March 2019; published 12 August 2019)

\begin{abstract}
Many popular attempts to explain the observed patterns of fermion masses involve a flavon field. Such weakly coupled scalar fields tend to dominate the energy density of the Universe before they decay. If the flavon decay happens close to the electroweak transition, the right-handed electrons stay out of equilibrium until the sphalerons shut off. We show that an asymmetry in the right-handed charged leptons produced in the decay of a flavon can explain the baryon asymmetry of the Universe.
\end{abstract}

DOI: 10.1103/PhysRevD.100.035011

\section{INTRODUCTION}

There is more matter than antimatter in the Universe. This baryon asymmetry of the Universe (BAU) is measured to be $[1,2]$

$$
\eta_{\mathrm{obs}} \equiv \frac{n_{B}-n_{\bar{B}}}{s} \simeq 8 \times 10^{-11},
$$

where $n_{B(\bar{B})}$ is the (anti)baryon number density and $s$ is the entropy density.

In order to explain the BAU, the three so called Sakharov conditions [3] must be satisfied: (i) baryon (or lepton) number must be violated, (ii) $C$ and $C P$ must be violated and (iii) there must be a departure from thermal equilibrium.

Baryon number and $C P$ are violated in the electroweak (EW) sector of the Standard Model (SM). However the $C P$ violation in the SM is too small to account for the BAU. In addition, there is no out-of-equilibrium process in the SM. In order to produce the BAU, beyond-the-SM physics with additional degrees of freedom (d.o.f.) is needed. These d.o.f. can also solve other deficiencies in the SM. The most notable example is in leptogenesis [4], where the right-handed neutrinos are the only required additional d.o.f. These right-handed neutrinos are part of the seesaw mechanism [5], and thus provide an explanation for the smallness of neutrino masses. Through their out-ofequilibrium decays, the observed BAU can be obtained.

In this paper, we show that another d.o.f., the flavon, that conceivably plays an important role in understanding fermion masses, can also generate the observed BAU. In general, a flavon is a scalar particle whose vacuum

Published by the American Physical Society under the terms of the Creative Commons Attribution 4.0 International license. Further distribution of this work must maintain attribution to the author(s) and the published article's title, journal citation, and DOI. Funded by SCOAP ${ }^{3}$. expectation value (VEV) dictates the (Yukawa) couplings of the SM fermions, with the most popular example being the Froggatt-Nielsen field [6]. We show that the out-ofequilibrium decays of such a flavon can be the necessary beyond-the-SM physics that generates the observed BAU. The flavon decays may create a left-right charged lepton asymmetry. In a universe where the flavon dominates the energy density, the right-handed electrons may not come into chemical equilibrium, and an asymmetry in these righthanded electrons can be converted into a baryon number asymmetry by sphalerons.

\section{BASIC MECHANISM}

Consider the couplings of the SM charged leptons to an SM singlet scalar, the flavon $S$, charged under the flavor symmetry $\mathrm{U}(1)_{\mathrm{FN}}$,

$$
\mathscr{L} \supset y_{0}^{f g}\left(\frac{v_{S}+S}{\Lambda}\right)^{n_{f g}} \bar{e}_{\mathrm{R}}^{g} \cdot \phi^{*} \cdot \ell_{\mathrm{L}}^{f}+\text { H.c. }
$$

Here, $\phi$ denotes the electroweak Higgs doublet, $\ell_{\mathrm{L}}^{f}$ is the $f$ th-generation left-handed lepton doublet, and $e_{\mathrm{R}}^{g}$ represents the $g$ th-generation right-handed charged lepton. $v_{S}$ is the flavon VEV, with $\Lambda$ being the cutoff scale of the flavor symmetry, $y_{0}$ an $\mathcal{O}(1)$ coupling constant and $n_{f g}$ integers related to the Froggatt-Nielsen charges under $\mathrm{U}(1)_{\mathrm{FN}}$. In the following, we will restrict ourselves to the simplest version of the scenario, in which the flavon only couples to leptons. As a benchmark model, we will take

$y_{0} \simeq 1, \quad \varepsilon=\frac{v_{S}}{\Lambda}=0.2, \quad n_{e}=9, \quad n_{\tau}=3$.

The key observation in our scenario is that the flavon decays

$$
S \rightarrow \bar{\ell}_{\mathrm{L}}+\phi+e_{\mathrm{R}}, \quad S^{*} \rightarrow \ell_{\mathrm{L}}+\phi^{*}+\bar{e}_{\mathrm{R}},
$$


violate left-right symmetry, e.g., an $S$ particle only decays into an antiparticle $\ell_{\mathrm{L}}$ and particle $e_{\mathrm{R}}$. Crucially, if there is an initial flavon asymmetry, say an excess of $S$ over $S^{*}$, after the flavons decay, there will be more left-handed antileptons than left-handed leptons. Note that the total lepton number, $L$, is conserved because the asymmetry in the left-handed leptons will be balanced by an equal and opposite asymmetry in the right-handed leptons. However, sphalerons will only act on left-handed leptons and partially convert this asymmetry into a baryon $(B)$ asymmetry. This is similar to Dirac leptogenesis [7], in which nonzero baryon number asymmetry is possible with $B-L$ conservation, as it utilizes the features that sphalerons only couple to left-handed fields. In the scenario discussed here, the left-right asymmetry originates from a primordial flavon-antiflavon asymmetry, which we will discuss below in more detail.

\section{FLAVON ASYMMETRY}

Our scenario requires the flavon to have a large asymmetry when it decays. This means that an asymmetry must be created and that flavon number must be conserved during the flavon oscillations. It has been pointed out in the context of the Affleck-Dine scenario [8] that scalar field dynamics in the early Universe can exhibit these features. As explained in Sec. III.C of [9], in supersymmetric theories the mass terms of scalar fields $S$ preserve $S$ number while interaction terms do not. These interactions may provide an "initial kick" (in the terminology of Ref. [10]) which leads to an angular momentum of the $S$ field in the complex plane, i.e., an $S$-number asymmetry. In general, if the lowest-order interactions preserve flavon number and the higher-order terms do not, the latter can lead to an initial asymmetry that is preserved when the higher-order terms become unimportant. While this has been discussed mostly in supersymmetric scenarios [9], such setups may also be a consequence of, say, a $\mathbb{Z}_{N}$ symmetry. As explicitly shown in Ref. [10], a large flavon asymmetry may originate from $S^{6} / \Lambda^{2}$ interactions if the flavon mass is of the order of the gravitino mass, $m_{S} \sim m_{3 / 2}$. For higher-dimensional terms, a large flavon asymmetry is even easier to produce [9]. That is, the Affleck-Dine mechanism provides us with a concrete example of a setting in which the large asymmetry is generated, yet there are other possibilities. The point of this paper, however, is that, due to the intricate dynamics of the chemical potential of the electrons and the sphaleron, an asymmetry of the $S$ particles, which carry neither baryon nor lepton number, can explain the baryon number of the Universe. Note that the higher-dimensional terms become unimportant later when the $S$ field performs coherent oscillations, such that the asymmetry persists until the flavon decays.

A reason for concern might be the origin of the $U(1)$ symmetry that ensures flavon number conservation. At first sight, one may conclude that if $\mathrm{U}(1)_{\mathrm{FN}}$ gets broken spontaneously, one is left with two options: (i) $\mathrm{U}(1)_{\mathrm{FN}}$ is local, in which case the would-be Goldstone mode gets eaten, and one is left with a real scalar and no $\mathrm{U}(1)_{S}$ that allows us to define the $S$-number; (ii) $\mathrm{U}(1)_{\mathrm{FN}}$ is global, in which case there is a Goldstone mode, and the situation is even worse. However, in explicit models the situation is often richer than that. For instance, in supersymmetric scenarios, the scalar fields are complex. In such models, one starts with more d.o.f. and the flavon is a complex linear combination of these fields, whose mass term preserves a U(1) symmetry. There are also nonsupersymmetric examples that have additional custodial symmetries (see e.g., Refs. [11,12] for recent applications of these symmetries in dark matter model building). We will assume that for energy scales far below $\Lambda$ an approximate $\mathrm{U}(1)_{S}$ is preserved by the flavon potential, i.e.,

$$
\mathscr{V}_{S}=m^{2}|S|^{2}+\left(\begin{array}{c}
S \text {-number-violating terms } \\
\text { suppressed by powers of } \Lambda
\end{array}\right) \text {. }
$$

\section{FLAVON COSMOLOGY}

By assumption, the flavon we consider is a weakly coupled scalar field. Hence it can perform coherent oscillations around the $T=0$ minimum of its potential. In fact, thermal corrections to the potential will push the flavon away from its expectation value at $T=0$ [13]. The energy density stored in these oscillations, $\rho_{S}$, only drops as $a^{-3}$ whereas the energy density of radiation, $\rho_{\text {rad }}$, drops as $a^{-4}$, where $a$ is the scale factor. It is expected that at a time $t_{*}$, corresponding to a temperature $T_{*}$, the energy density stored in flavon oscillations starts to dominate the radiation contribution. We define this point as

$$
\left.\rho_{S}\right|_{T=T_{*}}=\left.\rho_{\mathrm{rad}}\right|_{T=T_{*}} .
$$

The flavon decays to SM leptons with a decay rate

$$
\Gamma_{S} \sim \frac{1}{\varepsilon} \frac{\left|n_{\tau} y_{\tau}\right|^{2}}{64 \pi^{3}} \frac{m_{S}^{3}}{\Lambda^{2}} .
$$

Most of the decay products thermalize with the radiation and contribute to the radiation density. However, as we will show below, the right-handed electrons might not come into chemical equilibrium before the sphalerons have switched off.

The evolution of the relevant energy densities is given by

$$
\begin{gathered}
\frac{\mathrm{d} \rho_{S}}{\mathrm{~d} t}+3 H \rho_{S}=-\Gamma_{S} \rho_{S}, \\
\frac{\mathrm{d} \rho_{\mathrm{rad}}}{\mathrm{d} t}+4 H \rho_{\mathrm{rad}}=\Gamma_{S} \rho_{S},
\end{gathered}
$$


where the Hubble rate is determined by the Friedmann equation

$$
H^{2}=\frac{8 \pi}{3 M_{\mathrm{Pl}}^{2}}\left(\rho_{S}+\rho_{\mathrm{rad}}\right),
$$

with $M_{\mathrm{Pl}} \simeq 1.2 \times 10^{19} \mathrm{GeV}$ being the Planck mass.

We solve this set of equations numerically and show a benchmark scenario in Fig. 1. Analytical approximations for the two energy densities can be given at a time $t$ before the flavon decays, $t_{*}<t<\tau \sim \Gamma_{S}^{-1}$, as

$$
\begin{gathered}
\rho_{S}(t) \simeq \frac{M_{\mathrm{Pl}}^{2}}{6 \pi t^{2}} \mathrm{e}^{-\Gamma_{S} t}, \\
\rho_{\mathrm{rad}}(t) \simeq \frac{M_{\mathrm{Pl}}^{2} t_{*}^{2 / 3}}{6 \pi t^{8 / 3}}+\frac{\Gamma_{S} M_{\mathrm{Pl}}^{2}}{10 \pi t} .
\end{gathered}
$$

The first term in $\rho_{\text {rad }}$ is the initial radiation energy density and the second term is generated from flavon decays. This second term starts taking over $\rho_{\text {rad }}$ long before $\Gamma_{S}^{-1}$.

We will show in the next section that BAU can be produced via flavon decays for $\Gamma_{S} \sim O\left(10^{-13}-10^{-17} \mathrm{GeV}\right)$. Thus, using Eq. (7) we will require the flavon mass to be

$$
\frac{m_{S}}{\mathrm{TeV}} \sim\left(\frac{\Lambda}{10^{9} \mathrm{GeV}}\right)^{\frac{2}{3}}\left(\frac{\Gamma_{S}}{10^{-15} \mathrm{GeV}}\right)^{\frac{1}{3}}
$$

such that $\Lambda$ is below the Planck scale.

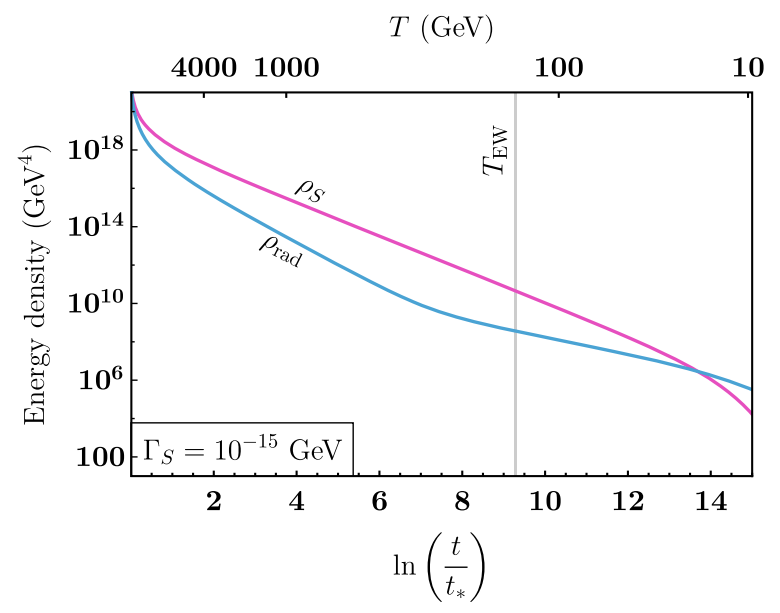

FIG. 1. Energy densities stored in flavon oscillations, $\rho_{S}$, and in radiation $\rho_{\text {rad }}$, with respect to temperature. As a benchmark scenario, it is assumed that $\rho_{S}$ starts to dominate at $T_{*}=$ $100 \mathrm{TeV}$ and the flavon decay rate is $\Gamma_{S}=10^{-15} \mathrm{GeV}$, which corresponds to a decay temperature $T_{\mathrm{d}} \simeq 10 \mathrm{GeV}$. We also show the temperature when the EW sphalerons shut off, $T_{\mathrm{EW}} \sim 160 \mathrm{GeV}$.

\section{GENERATION AND NONEQUILIBRATION OF RIGHT-HANDED ELECTRONS}

Among other leptons, the flavon decays into righthanded electrons with a branching fraction of $B_{e} \sim\left(\frac{n_{e} y_{e}}{n_{\tau} y_{\tau}}\right)^{2} \sim$ $7.5 \times 10^{-7}$. Through its decays, the flavon asymmetry will get partially converted into an asymmetry in right-handed electrons. Like in leptogenesis [4], this asymmetry is turned into a baryon asymmetry by sphalerons. Similarly to Dirac leptogenesis [7], our scenario does not require $B-L$ violation. However, our scenario works both for Dirac and Majorana neutrinos.

Right-handed electrons equilibrate with the SM plasma mainly through their interactions with the Higgs boson and $2 \rightarrow 2$ scatterings. This equilibration rate has been recently calculated to be [14]

$$
\Gamma_{\mathrm{LR}} \simeq 10^{-2} y_{e}^{2} T
$$

which is larger by almost an order of magnitude than the initial estimate [15] and by a factor of a few than a refined estimate [16]. Comparing this rate to the Hubble rate for a radiation-dominated universe, one finds that the righthanded electrons come into equilibrium at $T \sim 10^{5} \mathrm{GeV}$. Hence, in standard cosmology, any asymmetry in the righthanded electrons would be washed out long before the EW transition at $T \sim 160 \mathrm{GeV}$. However, in a universe that is dominated by a flavon until temperatures around the electroweak scale, right-handed electrons may not equilibrate. (See Fig. 2.)

We find the asymmetry in the number density of righthanded electrons after the flavon decays by solving the Boltzmann equation for $n_{\mathrm{R}}=n_{e_{\mathrm{R}}}-n_{\bar{e}_{\mathrm{R}}}$,

$$
\frac{\mathrm{d} n_{\mathrm{R}}}{\mathrm{d} t}=-3 H n_{\mathrm{R}}-\Gamma_{\mathrm{LR}} n_{\mathrm{R}}+B_{e} \Gamma_{S} n_{S}
$$

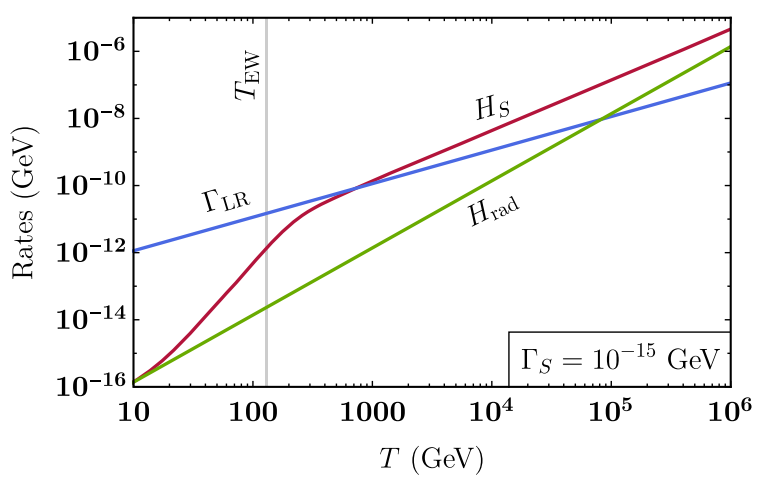

FIG. 2. Comparison of the equilibration rate of right-handed electrons, $\Gamma_{\mathrm{LR}}$, to the Hubble rates in a radiation-dominated universe, $H_{\mathrm{rad}}$ and in a universe with intermediate flavon domination, $H_{S}$. For this benchmark case the flavon energy density starts to dominate at $T_{*}=10^{7} \mathrm{TeV}$ and the flavon decays at $T_{\mathrm{d}} \simeq 10 \mathrm{GeV}$. 


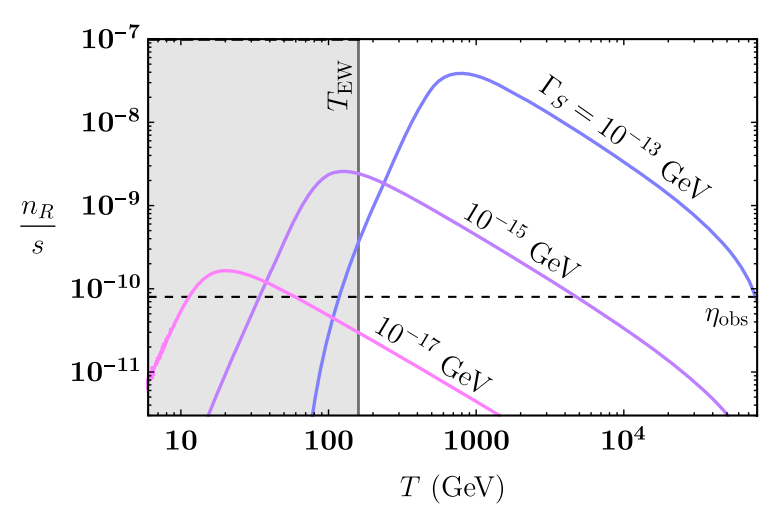

FIG. 3. Right-handed electron asymmetry generated through asymmetric flavon decays for different values of the flavon decay rate $\Gamma_{S}$. The observed baryon asymmetry $\eta_{\text {obs }} \simeq 8 \times 10^{-11}$ is shown for reference.

where $B_{e}$ is the flavon branching ratio to electrons. The asymmetry in the flavon number density, $n_{S}$, is parametrized through an initial flavon asymmetry, $\eta_{S}$, as

$$
n_{S} \equiv \eta_{S} \frac{\rho_{S}}{m_{S}}
$$

We solve for $n_{\mathrm{R}}$ together with Eq. (8). In Fig. 3 we show the dimensionless quantity $\eta_{\mathrm{R}}=\frac{n_{\mathrm{R}}}{s}$, where $s$ is the entropy density, for a benchmark scenario with

$$
m_{S}=1 \mathrm{TeV}, \quad \eta_{S}=1, \quad T_{*}=100 \mathrm{TeV} .
$$

As usual, the temperature is defined as the temperature of radiation, which may or may not dominate the energy density of the Universe.

The behavior of $\eta_{R}$ can be understood qualitatively as follows. A right-handed electron asymmetry is produced through flavon decays and is proportional to the flavon number density. When the Hubble rate drops below the leftright equilibration rate, the asymmetry is washed out by SM interactions. The time when this washout starts depends on the flavon lifetime, $\tau=\Gamma_{S}^{-1}$, and is generally before $\tau$. A smaller $\Gamma_{S}$ can delay the equilibration until after sphalerons shut off at $T_{\mathrm{EW}} \sim 160 \mathrm{GeV}$. However, this also means that a smaller number of flavons decay before $T_{\mathrm{EW}}$. Hence, in general, there is a balance between the flavon lifetime and $\Gamma_{\mathrm{LR}}$ that describes the region where the right amount of right-handed electron asymmetry is produced before the sphalerons shut off.

\section{BARYON ASYMMETRY}

In the SM $B+L$ number is violated by $\mathrm{EW}$ processes called sphalerons $[17,18]$. Sphalerons are unsuppressed above the EW transition temperature $T_{\mathrm{EW}}$, with a thermal rate $\Gamma_{\mathrm{sph}} \simeq\left(\alpha_{2} T\right)^{4}$, while they are exponentially suppressed due to finite gauge boson masses after EW symmetry

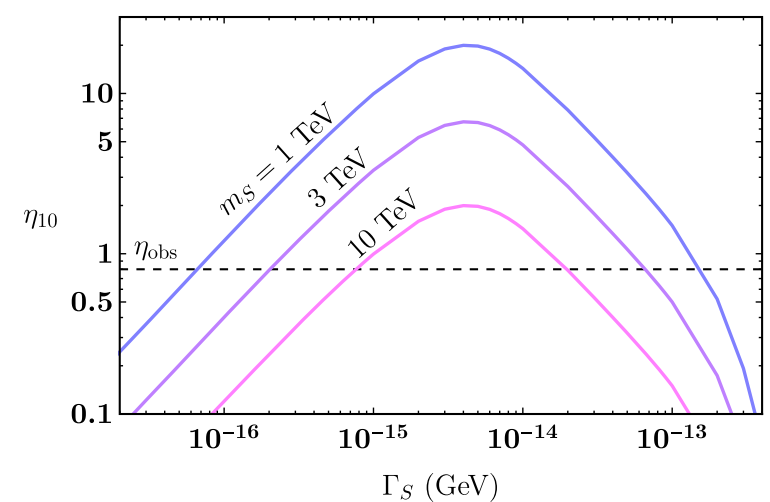

FIG. 4. Baryon asymmetry $\left(\eta_{10} \equiv \eta_{B} \times 10^{10}\right)$ of the Universe as a function of the flavon decay rate $\Gamma_{S}$ for different values of the flavon mass $m_{S}$. As a benchmark scenario we take the initial flavon asymmetry $\eta_{S}=1$. The observed baryon asymmetry $\eta_{\mathrm{obs}} \simeq 8 \times 10^{-11}$ is shown for reference.

breaking. While sphalerons violate $B+L$, they conserve $B-L$. Hence a lepton asymmetry will be turned into a baryon asymmetry [4]. In our scenario this baryon asymmetry is given by [16]

$$
\left.\eta_{B} \equiv \frac{n_{B}}{s} \simeq \frac{198}{481} \frac{n_{\mathrm{R}}}{s}\right|_{T_{\mathrm{EW}}} .
$$

We numerically solve Eqs. (8) and (13) to find the electron asymmetry and the entropy of the Universe at $T_{\mathrm{EW}} \simeq 160 \mathrm{GeV}$. Our results are shown in Figs. 3 and 4. As can be seen in Fig. 4, there is a large parameter space where asymmetric flavon decays produce the observed BAU. We comment on the dependence of $\eta_{B}$ on the parameters of the model.

(1) Our scenario is rather sensitive to the flavon decay rate. If the flavon decays too early, $T_{\mathrm{d}} \gg T_{\mathrm{EW}}$, righthanded electrons equilibrate and (essentially) no baryon asymmetry is generated. If the flavon decays too late, $T_{\mathrm{d}} \ll T_{\mathrm{EW}}$, the right-handed electron asymmetry is produced when sphalerons are inoperative, and again no baryon asymmetry emerges. We find that the observed baryon asymmetry is produced for $10^{-16} \mathrm{GeV} \lesssim \Gamma_{S} \lesssim 10^{-13} \mathrm{GeV}$.

(2) The baryon asymmetry is virtually independent of the time $t_{*}$ when the flavon domination starts as long as $T_{*} \gg T_{\mathrm{d}}, T_{\mathrm{EW}}$.

(3) As the flavon mass increases, its number density drops. This is because each flavon decay produces entropy, with the dilution factor being $m_{S} / T_{\mathrm{d}}$. Hence the baryon asymmetry is inversely proportional to $m_{S}$. The flavon decay rate also depends on its mass. For given values of $m_{S}$ and $\Gamma_{S}$, the appropriate flavon scale $\Lambda$ can be found from Eq. (11). We find that a flavon with $\mathcal{O}(\mathrm{TeV})$ mass can produce the observed BAU. This requires 
$\Lambda \sim \mathcal{O}\left(10^{9} \mathrm{GeV}\right)$. We note that for such a large flavon scale, constraints on the flavon mass are $\mathcal{O}(100 \mathrm{GeV})$ [19].

(4) The baryon asymmetry is directly proportional to the primordial flavon asymmetry $\eta_{S}$. We show our results in Fig. 4 for the maximal value of $\eta_{S}=1$. If we allow for a flavon as light as $200 \mathrm{GeV}$, we only require $\eta_{S} \gtrsim 10^{-2}$.

\section{CONCLUSIONS}

In this paper, we have shown that the observed baryon asymmetry can be produced in flavon decays. This scenario requires the flavon to decay around the time of the electroweak transition and that the flavon carries an $\mathcal{O}\left(10^{-2}-1\right)$ primordial asymmetry. The flavon mass is $\mathcal{O}(1-10 \mathrm{TeV})$. The viable parameter ranges are illustrated in Fig. 4. The role of the flavon is twofold:

(1) Its decays produce a left-right asymmetry in the lepton sector, the left-handed part of which is converted to a baryon asymmetry by sphalerons.

(2) It dominates the Universe before the EW scale, thus increasing the Hubble rate and preventing the righthanded electrons from equilibrating.
Our scenario is rather constrained and thus predictive. We "lose" a significant amount of efficiency because our scalar is a flavon, i.e., it predominantly decays into the heavier generations. A more "efficient" version of this scenario would be to couple a scalar to light fermions only, in which case one may also consider the decay into up or even down quarks. Note that this scalar does not need to be the flavon, but any complex scalar field that satisfies the above properties could give these results. However, it is arguably an appealing aspect of our scenario that we do not have to introduce a new d.o.f. in order to explain the observed baryon asymmetry. Rather, a traditional flavon can do that without being tweaked.

\section{ACKNOWLEDGMENTS}

This work is supported in part by NSF Grant No. PHY1620638 and by NSF Grant No. PHY-1719438. S. I. acknowledges support from the UC Office of the President via a UC Presidential Postdoctoral fellowship. This work was performed in part at Aspen Center for Physics, which is supported by National Science Foundation Grant No. PHY-1607611.
[1] P. A. R. Ade et al. (Planck Collaboration), Astron. Astrophys. 594, A13 (2016).

[2] S. Riemer-Sørensen and E. S. Jenssen, Universe 3, 44 (2017).

[3] A. D. Sakharov, Pis'ma Zh. Eksp. Teor. Fiz. 5, 32 (1967) [Usp. Fiz. Nauk 161, 61 (1991)].

[4] M. Fukugita and T. Yanagida, Phys. Lett. B 174, 45 (1986).

[5] P. Minkowski, Phys. Lett. 67B, 421 (1977).

[6] C. D. Froggatt and H. B. Nielsen, Nucl. Phys. B147, 277 (1979).

[7] K. Dick, M. Lindner, M. Ratz, and D. Wright, Phys. Rev. Lett. 84, 4039 (2000).

[8] I. Affleck and M. Dine, Nucl. Phys. B249, 361 (1985).

[9] M. Dine and A. Kusenko, Rev. Mod. Phys. 76, 1 (2003).

[10] R. Kitano, H. Murayama, and M. Ratz, Phys. Lett. B 669 , 145 (2008).

[11] T. Hambye, J. High Energy Phys. 01 (2009) 028.
[12] G. Arcadi, C. Gross, O. Lebedev, Y. Mambrini, S. Pokorski, and T. Toma, J. High Energy Phys. 12 (2016) 081.

[13] B. Lillard, M. Ratz, M. P. Tait, Tim, and S. Trojanowski, J. Cosmol. Astropart. Phys. 07 (2018) 056.

[14] D. Bödeker and D. Schröder, J. Cosmol. Astropart. Phys. 05 (2019) 010.

[15] B. A. Campbell, S. Davidson, J. R. Ellis, and K. A. Olive, Phys. Lett. B 297, 118 (1992).

[16] J. M. Cline, K. Kainulainen, and K. A. Olive, Phys. Rev. D 49, 6394 (1994).

[17] F. R. Klinkhamer and N. S. Manton, Phys. Rev. D 30, 2212 (1984).

[18] V. A. Kuzmin, V. A. Rubakov, and M. E. Shaposhnikov, Phys. Lett. 155B, 36 (1985).

[19] M. Bauer, T. Schell, and T. Plehn, Phys. Rev. D 94, 056003 (2016). 